\title{
Spatial interpolation of point velocities in stream cross-section
}

\author{
Eliška Hasníková*, Jiř́i Pavlásek, Marek Vach \\ Faculty of Environmental Sciences, Czech University of Life Sciences Prague, Kamýcká 129, Praha 6 - Suchdol, 165 21, Czech Republic. \\ *Corresponding author. Tel.: +420732371341. E-mail: hasnikova@fzp.czu.cz
}

\begin{abstract}
The most frequently used instrument for measuring velocity distribution in the cross-section of small rivers is the propeller-type current meter. Output of measuring using this instrument is point data of a tiny bulk. Spatial interpolation of measured data should produce a dense velocity profile, which is not available from the measuring itself. This paper describes the preparation of interpolation models.

Measuring campaign was realized to obtain operational data. It took place on real streams with different velocity distributions. Seven data sets were obtained from four cross-sections varying in the number of measuring points, 24-82. Following methods of interpolation of the data were used in the same context: methods of geometric interpolation arithmetic mean and inverse distance weighted, the method of fitting the trend to the data thin-plate spline and the geostatistical method of ordinary kriging. Calibration of interpolation models carried out in the computational program Scilab is presented. The models were tested with error criteria by cross-validation. Ordinary kriging was proposed to be the most suitable interpolation method, giving the lowest values of used error criteria among the rest of the interpolation methods.
\end{abstract}

Keywords: Open channel; Current meter; Cross-validation; Arithmetic mean; Inverse distance weighted; Thin-plate spline; Kriging.

\section{INTRODUCTION}

A propeller-type current meter is frequently used measurement instrument for point velocity of open channels in the Czech Republic, especially streams. The number of revolutions of the current propeller is proportional to the velocity in the particular place of the river cross-section. The propeller-type current meter as the most affordable instrument has the disadvantage of scatterness of the data that is the output of the measurement. In order to produce a dense cloud of data measurement would have to take many hours, which is not realizable in practice. River discharge is usually the output of processing velocity data. Routine processing by Harlacher method or by the method of vertical section segments results from a velocity information from cross-section verticals, being specified by standards (e.g. ČSN EN ISO 748, 2008 in the Czech Republic). Working with complex information, not only the vertical ones, requires a spatial procedure.

This paper presents the approach of assimilation of velocity vectors magnitude only, which is often the approach used in the hydrometrical computing. Geospatial types of the interpolation are commonly used to produce the shape of terrain in geography, to estimate the precipitation in hydrology, to estimate the deposits of ore in geology or to define the soil characteristics in pedology. Why not use them also in the task of processing the velocity profile of river cross-section? A spatial interpolation can provide information about the magnitude of the velocity in more points, where it was not measured. Knowing the velocity distribution in the river is helpful especially in those parts, where the sediment transport can play a role.

Software application HYDROS by Starý, used as a standard in Czech hydrometeorological institute (Starý, 2012), uses cubic spline to define velocity distribution in cross-section. The cubic spline fit between the points in the verticals is made at first, the cubic spline fit between the verticals in horizontal direction is made after. The same principle, but with polynomial regression instead of cubic spline, was successfully used in the paper of Stošić et al. (2012). Real spatial approach was realized by Ünal and Özcakal (2011) by Surfer 8.0 software. They separately interpolated two data sets by ten methods implemented in the program and pointed triangulation with linear interpolation, natural neighbour and spline as the best methods.

In presented paper, it was chosen four following methods and computation was done in the free and open source software Scilab (Baudin and Consortium Scilab - DIGITEO, 2011). Arithmetic mean is the simplest method of the local exact interpolations. It should be giving the most straightforward estimation of velocities, because its formula does not include any additional and possibly confusing coefficients. The arithmetic mean and the inverse distance weighted interpolation (IDW) are computationally much more inexpensive compared to two other chosen methods. Thin-plate spline - a spatial analogy of one-dimensional cubic spline, was chosen, because the mentioned studies had good results by spline. Ordinary kriging was chosen, as it is said to produce the best linear unbiased estimation (Clark, 2001; Webster and Oliver, 2007; and others). Spatial interpolation models well calibrated with a view of hydraulic laws, which is not covered by any geographical software, should be the tool for the estimation of velocity distribution of a stream cross-section as well as the tool for evaluation of stream discharge. The aims of this paper are the evaluation of velocity distribution using the selected interpolation methods and the comparison of the methods applied on measured point velocities in a stream crosssection.

\section{METHODS AND MATERIALS Measuring the data}

Four cross-sections of three streams were chosen for measuring. Reasons for the choice were the difference in shape and roughness of measuring profiles - natural and enrocked mandesigned stream bed, suitable discharge and geometry of cross-sections.

A steel tape was used for tracing cross-sections and evaluating the distances between gauging verticals. A propellertype current meter has being set step by step on a wading rod to 


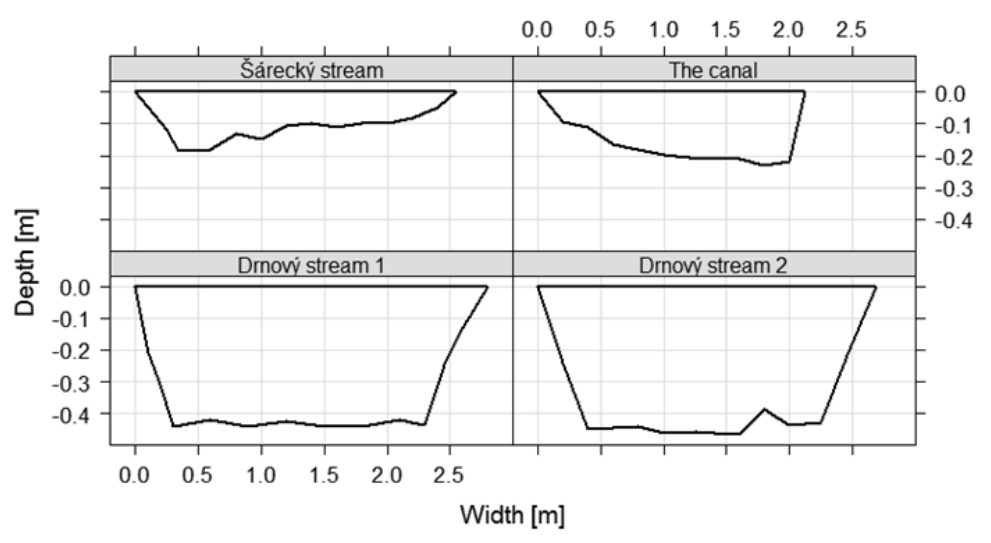

Fig. 1. Four measured cross-sections from the viewpoint upstream.

the gauging verticals. Three different measuring units of OTTC-2 on the wading rods were used, the same propeller was used for the whole cross-section within each measurement of crosssections. Measuring time in each point was 30 seconds, measured by OTT signal counter. The measurements were recorded on paper hydrometric sheets. The measuring campaign was held in April and August 2010 and 2011.

There was a steady flow in the course of all measurements. In total, seven available data sets were collected by the measurements. The cross-sections were less than $3 \mathrm{~m}$ wide, their maximum depth was under $0.5 \mathrm{~m}$ and on average 60 particular measuring points were measured in each crosssection. The placement of the points in the gauging verticals was according to hydrometric conventions (CSN EN ISO 748, 2008). Depending on the number of particular points in the vertical, their position was: close to the stream bed $(0.05 \mathrm{~m}$ from the stream bed), in $0.2,0.4,0.6$ and 0.8 times the depth and in the immediate vicinity to the water surface. Selected cross-sections were with the normal velocity distribution. To obtain more variety in velocity distributions, a barrier was put up on the bottom of the stream bed in front of the measured cross-section for three measurements - a block of two layers of two firebricks. The view of four cross-sections from upstream is in Fig. 1.

First measurement was realized on Šárecký stream in downstream part of Šrecké valley in Prague. The cross-section was without bed vegetation with stationary pebbly bed, and was of an asymmetric shape. The top width of the cross-section was $2.55 \mathrm{~m}$, the range of depths in gauging verticals was 0.08 $0.18 \mathrm{~m}$.

Second measurement was realized on Drnový stream in Klatovy. Drnový stream is regulated and flows in a symmetrical trapezoidal river channel. The river-basin is composed of a grass in an upper part and an enrockment in a lower and bottom part - while measuring, water-level was just in the lower part. The measurements were realized on two cross-sections on Drnový stream. First cross-section had these characteristics: the top width of $2.8-2.82 \mathrm{~m}$, the bottom width of $2 \mathrm{~m}$, the maximum depth of 41-44 m, the side slopes 1:0.68 and 1:1.15. Second cross-section had these characteristics: the top width $2.65-2.68$, the bottom width of $1.85 \mathrm{~m}$, the maximum depth of $0.44-0.465$, the side slopes 1:0.89 and 1:1.

Last measurement took place on the north edge of Rohozno on the canal, joining the stream Korytský potok with the river Úhlava. The canal had an asymmetric shape with partly muddy bottom with remains of vegetation matters from streamside stand. The top width of the cross-section was $2.12 \mathrm{~m}$, the range of depths in gauging verticals was $0.1-0.23 \mathrm{~m}$.

\section{Interpolation methods used}

Each estimation model was composed of one of four exact methods. These methods were arithmetic mean and inverse distance weighted (IDW), as two geometrical interpolation methods, thin-plate spline, as a method of fitting the trend to the data of surface, and the geostatistical method of ordinary kriging. In the exact interpolations, an estimated velocity in required point is a function of known velocities in neighbouring measured points. Weights are assigned to the neighbouring points and the sum of the weights equals one. A velocity $\boldsymbol{v}_{\boldsymbol{0}}$ in a point $x_{0}$ in space is estimated by the linear estimation $\boldsymbol{v}_{0}=$ $\hat{\boldsymbol{v}}\left(x_{0}\right)$ as (1). Minimal difference between estimated and true value in a point $\boldsymbol{x}_{\mathbf{0}}$ is required (Fedorov, 1987).

$\hat{\boldsymbol{v}}\left(x_{0}\right)=\boldsymbol{w}^{T} \boldsymbol{v}$,

where $\boldsymbol{w}^{\boldsymbol{T}}=$ transposed vector of weights $\boldsymbol{w} \in R^{n}, \boldsymbol{v}=$ $\left(v_{1}, v_{2}, \ldots, v_{n}\right)$, where $n$ is the number of neighbouring points, $v_{i}=$ $v\left(x_{i}\right)$.

Estimation of the velocity in a point $x_{0}$ by the equation of arithmetic mean assumes the same weight of all neighbouring points - the inverse value of the number of neighbouring points, no spatial correlation between data locations (Wackernagel, 2003).

The method of IDW counts with the inverse distance between a neighbouring measured point $\boldsymbol{x}_{\boldsymbol{i}}$ and an estimated point $x_{0}$ (2). More distant measured point has smaller value of weight than less distant point.

$\hat{\boldsymbol{v}}\left(x_{0}\right)=\frac{\sum_{i=1}^{n} d_{0, i}^{-\beta} \boldsymbol{v}\left(x_{i}\right)}{\sum_{i=1}^{n} d_{0, i}^{-\beta}}$

where $n$ denotes the number of neighbouring points, $\beta=$ integer constant usually up to $5, d_{0, i}=\sqrt{\left(X_{0}-X_{i}\right)^{2}+\left(Z_{0}-Z_{i}\right)^{2}}$. $X_{0}$ and $Z_{0}$ represent $\mathrm{x}$ and $\mathrm{z}$-coordinates of a point $x_{0}$ and analogically for a point $x_{i}$. 
The third method used was spatial cubic spline, namely thinplate spline. Estimation of velocity follows Dyn et al. (1986)

$\hat{\boldsymbol{v}}\left(x_{0}\right)=P\left(x_{0}\right)+\sum_{i=1}^{n} w_{i} \varphi_{i}\left(d_{0, i}\right)$

where $n$ is number of neighbouring points, $\varphi\left(d_{0, i}\right)$ is a vector of radial basis functions

$\varphi\left(d_{0, i}\right)= \begin{cases}d_{0, i}^{2} \ln d_{0, i}, & d>0 \\ 0, & d=0\end{cases}$

with distance $d_{0, i}$ of point $x_{0}$ and points $x_{i}$ as

$d_{0, i}=\sqrt{\left(X_{0}-X_{i}\right)^{2}+\left(Z_{0}-Z_{i}\right)^{2}}$.

And polynomial $P\left(x_{0}\right)=a_{1}+a_{2} X_{0}+a_{3} Z_{0}$ for known coordinates $\left[X_{0}, Z_{0}\right]$ of estimated point $x_{0}$ (Bookstein, 1989). Weights $w_{i}$ and coefficients $a_{j}$ were computed according to Bookstein (1989).

An initial step using kriging is computing an experimental variogram (in general form 4), which represents the variances of differences between measured values within the lag.

$2 \gamma *(h)=\frac{1}{n} \sum_{i=1}^{n}\left[v\left(x_{i}\right)-v\left(x_{i}+h\right)\right]^{2}$

where * denotes „experimental“" value of the variogram calculated from measured values, $n$ is the number of measured points, $\boldsymbol{v}\left(x_{i}\right)=$ the velocity in a position $x_{i}, \boldsymbol{v}\left(x_{i}+h\right)=$ the velocity in the lag distance $h$ from velocity in a position $x_{i}$ (Clark, 2001).

Half the variogram represents the semivariogram. Value of the experimental semivariogram and the distance between points in experimental semivariogram are variables, on which the selection of a theoretical model of semivariogram depends. Four theoretical models were considered in this case: linear, exponential, spherical and Gaussian model (Webster and Oliver, 2007). The omnidirectional semivariogram, as well as the semivariograms of horizontal and vertical directions were considered. Weights of neighbouring points to be filled into (1) are calculated using ordinary kriging according to (5) (Raid et al., 2013)

$\left(\begin{array}{l}\boldsymbol{w} \\ \lambda\end{array}\right)=\left(\begin{array}{ll}\boldsymbol{\gamma}\left(S_{i}, S_{j}\right) & \boldsymbol{J} \\ \boldsymbol{J}^{T} & 0\end{array}\right)^{-1}\left(\begin{array}{l}\gamma\left(S_{i}, V\right) \\ 1\end{array}\right)$

where $\lambda=$ Lagrange multiplier, $\boldsymbol{J}$ and $\boldsymbol{J}^{\boldsymbol{T}}=$ unit vectors, $\boldsymbol{w}^{\boldsymbol{T}}=$ $\left(w_{1}, w_{2}, \ldots, w_{n}\right)$ is transposed vector of weights $w_{i}, \gamma\left(S_{i}, V\right)$ are covariances of samples $S_{i}$ and volume to be estimated $V$, $\gamma\left(S_{i}, S_{j}\right)$ are covariances of samples $S_{i}$ and samples $S_{j}$.

To follow hydraulic laws, as behaviour of fluid near the stream walls belongs to a transition region between boundary layer and turbulent layer conditions, following assumption was set. Velocity is assumed to be zero on the tops of friction patterns, interpolation by simple power expansion is used between the zone of the tops of friction patterns and the zone of lowest measured points in the verticals.

\section{Parameters of calibration}

A program for numerical computing Scilab, version 5.3.3, was used for computations of a calibration and a validation. Basic build-in functions of the program were used and own scripts were produced, no toolbox was used. Four measurement series were selected as calibration data sets, three were used for validation. A verification processes were mostly based on the cross-validation principle. One different measuring point was dropped in each calibration calculation and a value of velocity was calculated there. This means that the calibration through the cross-validation had following number of loops: the number of measured points in all calibration data sets multiplied by the number of combinations of tested parameters.

The tested calibration parameters were:

1. the parameters of the interpolation formulas,

2. a type of ,coordinate system“,

3. a shape of neighbourhood,

4. boundary conditions,

5. a special condition.

Parameter $\beta$ from IDW formula (2) was tested for values $[1 ; 2 ; 3]$. The number of points used in the interpolation, which is denoted by $n$ in the models, was selected from integers in an interval

$\langle 1$; number of points with known velocity in cross-section $\rangle$.

\section{Zero z-coordinate in the water surface}

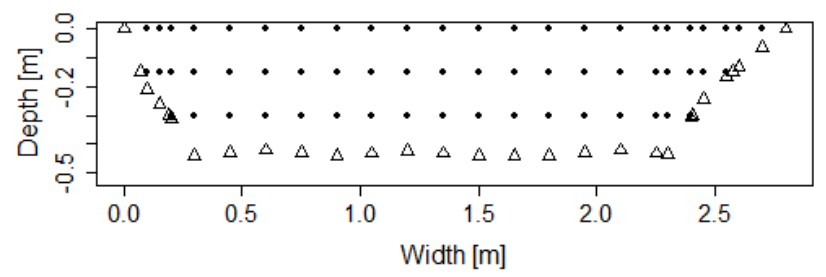

Zero z-coordinate in the stream bed

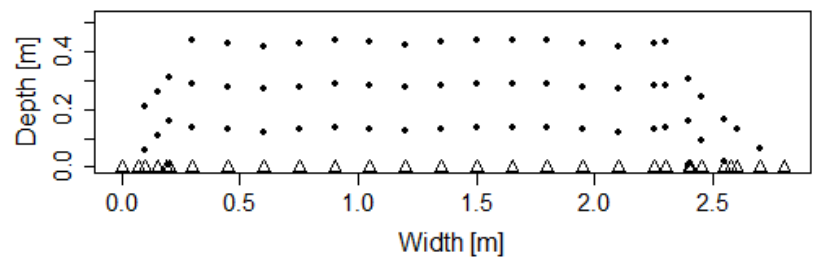

- Mesh - the inner part $\Delta$ Mesh - the stream bed

Fig. 2. The orthogonal mesh in a cross-section visualised in two types of coordinate system, that were used as one of parameters in the calibration process.

„Coordinate system“ was of two types with a difference in zcoordinate (vertical coordinate). One with zero in the stream bed and positive values rising towards the water surface, second with zero on the water surface and with negative values towards the stream bed (Fig. 2).

The neighbourhood of each calculated point was tested in the shape of ellipse with major axis parallel to the water surface. The elliptical shape should ensure that the interpolations are not based only on the values in their own vertical, as opposed to most other methods of measuring discharge evaluation. The ratio of major and minor axis $a: b$ was gradually increased. The 
value of minor axis $b$ stayed 1 and the value of major axis $a$ was chosen from the set $\langle 1,1.01,1.02,1.03 \ldots 5\rangle$.

The boundary conditions were based on hydraulic presumptions, three types were tested separately:

a. no boundary condition,

b. zero point velocity in grid nodes of intersections of a stream bed with gauging verticals,

c. zero point velocity in grid nodes of intersections of a stream bed with gauging verticals; point velocity in an intersection of a gauging vertical with a water surface assumed equal to velocity measured in a closest position to a water surface in a gauging vertical.

The special condition was established by ignoring the information from one's own vertical. This condition should have similar effect as setting an elliptic neighbourhood.

\section{Calibration and evaluation of outputs}

Error criteria, error graphs, which show measured values against estimated ones, and visualization of the simulated points and the measured points together were used for determining the better models. The error criteria used were the mean error (ME), the mean absolute error (MAE), the root mean squared error (RMSE) and the standard deviation of residuals (STD), in their relative form for comparison between the data sets and in absolute form. The substitution of the difference between the measured and estimated velocity $\boldsymbol{v}\left(x_{i}\right)-\hat{\boldsymbol{v}}\left(x_{i}\right)$ with the fraction $\frac{\boldsymbol{v}\left(x_{i}\right)-\hat{\boldsymbol{v}}\left(x_{i}\right)}{\boldsymbol{v}\left(x_{i}\right)}$ in the equations of the absolute form of the criteria (Madsen et al., 2010) was done to obtain the relative form of the criteria equations.

RMSE and MAE were found for every measured/estimated point and the arithmetic means of both criteria were calculated for the calibration data sets. A model with a minimal value of the arithmetic means of these criteria was stated to be the model with the best parameters. The mentioned approach was applied for arithmetic mean and IDW, a different approach was applied for the calibration of thin-plate spline and kriging. The minimum approach was found not appropriate for thin-plate spline in all situations. The results of thin-plate spline were judged separately for each calibration data set. Kriging also had a different approach. Fitting a semivariogram to the calibration data was done to each cross-section data set to calibrate universal kriging model for all measured data sets. Two theoretical semivariogram models were found the best and RMSE and MAE were calculated for all cross-section data sets for this method. After the selection of the semivariogram model, the rest of model parameters were tested on the same calibration data sets in the same way as the other methods.

The calibration of the data led to the definition of the parameters of the interpolation formulas. The best parameters were used for the validation of chosen interpolation models. The same error criteria as for the calibration and the error graphs were used also for the validation part. Significance of the difference between measured and estimated values of validation data sets was measured by pair t-test in the statistical software R, version 3.0.2 (Venables et al., 2014). Unpaired test was used to evaluate the difference between the mean of the measured values and the estimated ones. An association between MAE and measured velocities, between MAE and distance of a point's location from a bank and between MAE and depth of a point's location was tested by Pearson's correlation coefficient in the statistical software R, too.

\section{RESULTS \\ Models of interpolation}

The best number of neighbouring points used for the estimation using the arithmetic mean was detected number 3 . The relative value of the mean RMSE of all calibration data sets changing with the number of neighbouring points is shown in Fig. 3. Another graph in the same Figure represents the changing value of RMSE with the different rate of minor and major axes of the elliptic neighbourhood. The lowest value of RMSE criterion was detected for three known values in the neighbourhood of the elliptic shape with the scale of major and minor axis $a: b=1.6: 1$. Other parameters chosen as better of the tested ones were z-coordinate with zero value in the stream bed and the boundary condition labelled as c. in the part of Parameters of calibration.

The best interpolation by IDW was made in the coordinate system with zero value of z-coordinate in the stream bed. A boundary condition was settled as c. from Parameters of calibration of this paper. The model of interpolation (6) gave the best results with the estimation based on twenty known points (Fig. 3 ) from the elliptic neighbourhood with the scale of major and minor axis $a: b=1: 1$ (Fig. 3). Parameter $\beta$ set on value 1 gave the best error value (RMSE in the relative form $=0.229$ ), as shown in Fig. 3.

$\hat{\boldsymbol{v}}\left(x_{0}\right)=\frac{\sum_{i=1}^{20} d_{i}^{-1} \boldsymbol{v}\left(x_{i}\right)}{\sum_{i=1}^{20} d_{i}^{-1}}$

No particular value $n$ of known points was found for the method of thin-plate spline, the formula (3). The criteria mean (example of RMSE in Fig. 3) showed an inconsistent picture, each data set showed different preferred value. But a linear relation of the number of points used for the interpolation, denoted by $n$, with the total number of measured points, denoted by $m$, was found (7).

$n=-0.07 \cdot m+8.5$

No boundary condition was found the best. The elliptic neighbourhood with the scale of major and minor axis of $a: b=2.8: 1$ was chosen (Fig. 3) and the coordinate system with zero value of $\mathrm{z}$-coordinate at the bottom.

An omnidirectional semivariogram of Gauss type was selected up by the „by eye“ method as the best semivariogram model of the ordinary kriging

$\gamma(h)=0.003+0.035 \cdot\left(1-\exp \left(-\frac{h^{2}}{0.38^{2}}\right)\right)$

where $\gamma$ is semivariogram function of the lag distance $h$.

The omnidirectional semivariogram means a rounded shape of a neighbourhood of an estimated point. The cross-validation had the best results with $z$-coordinate with zero in the water surface, provided there is no boundary condition and the number of neighbouring points for the purpose of the estimation $n=$ 3. Fig. 3 shows the growth of the relative RMSE criterion by one used point, but when error graphs are considered, better results were registered for three neighbours. 
a) Shape of neighbourhood

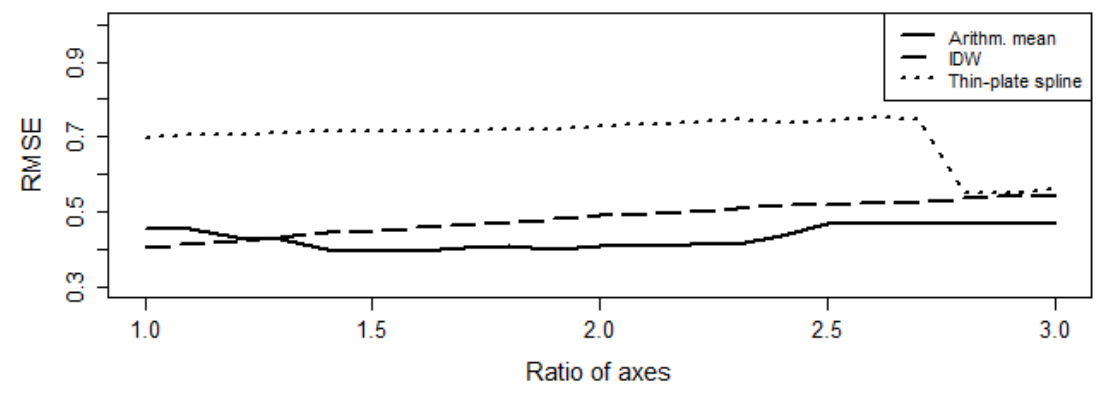

b) Number of values to make estimation

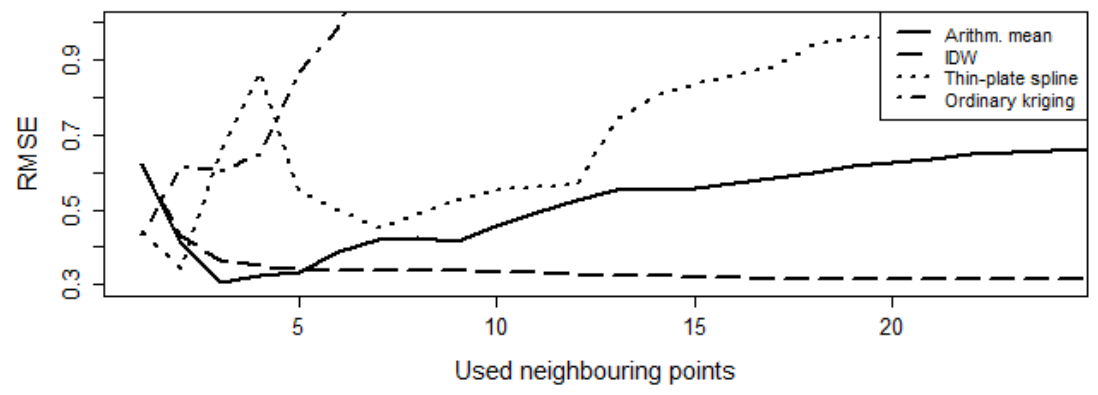

c) Beta parameter of Iverse distance weighted

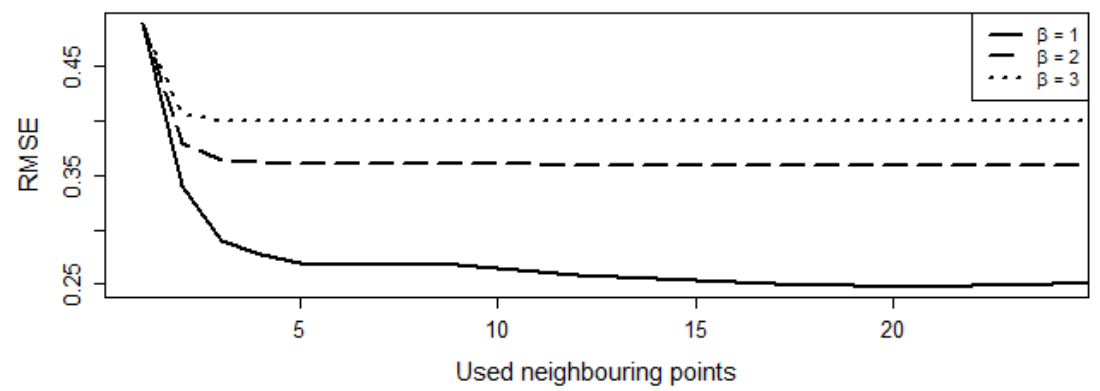

Fig. 3. Mean relative value of RMSE for the calibration data sets. a) RMSE in relation with the ratio of axes for three interpolation methods, b) RMSE in relation with the number of neighbouring points used in estimation for all methods, c) RMSE in relation with the number of neighbouring points for IDW with different values of parameter $\beta$.

\section{Value of errors and velocity profile}

The difference between values of computed criteria for the calibration data sets and the validation data sets on the final interpolation models was similar for the arithmetic mean, IDW and the thin-plate spline. As far as RMSE is considered, the criterion had about $53 \%$ higher value for the validation data sets then for the calibration data sets for the arithmetic mean, about $59 \%$ higher for IDW, $61 \%$ for the thin-plate spline and $2 \%$ for the ordinary kriging. The minimum values of majority of absolute and relative criteria were reached by the arithmetic mean (Table 1), maximum values were obtained by the thinplate spline. The mean value of ME being minimum for the ordinary kriging, but not for MAE (Table 1) shows a symmetrical distribution of overestimated and underestimated velocities by the method.

A distribution of MAE along a cross-section is in Fig. 4, where the validation data set 1 is used as an example. The arithmetic mean generated underestimation of lower velocities (Fig. 4). The IDW method showed the highest error rate of all methods. The whole lower half of the cross-section showed errors. The velocities are underestimated there, too. The ordinary kriging made an overestimation of velocities close to the stream bed. No linear dependence of MAE on velocity was found for any method, weak positive relationship with distance from the banks was found for all methods. MAE by the arithmetic mean and IDW were in positive moderate relationship with the depth (higher depth higher error), Pearson's $r$ for the arithmetic mean was 0.29 and for IDW was 0.38 .

Interpolation using IDW produces triangular forms representing so-called ,bull's eyes“ (Fig. 5) that do not occur in a real velocity profile with high probability. The ordinary kriging and the thin-plate spline produced rather monotone profile in comparison with the arithmetic mean and IDW (Fig. 5).

The estimated velocities were significantly different (confidence level 0.95) from the measured ones by all methods except the ordinary kriging. The mean of estimated velocities and the mean of measured velocities were not significantly different (confidence level 0.95) for any of the methods. The mean velocity closest to the mean of measured values was calculated from the estimations by the ordinary kriging (Table 2). 


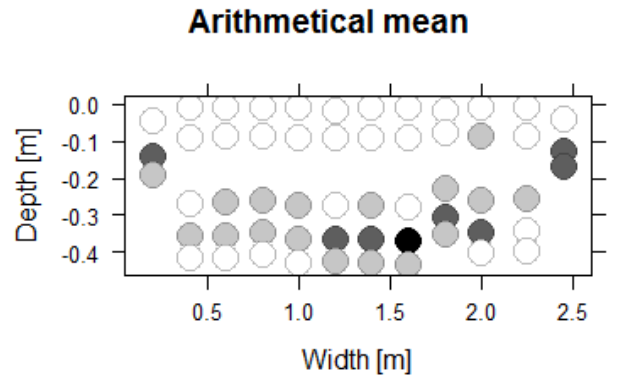

Thin-plate spline

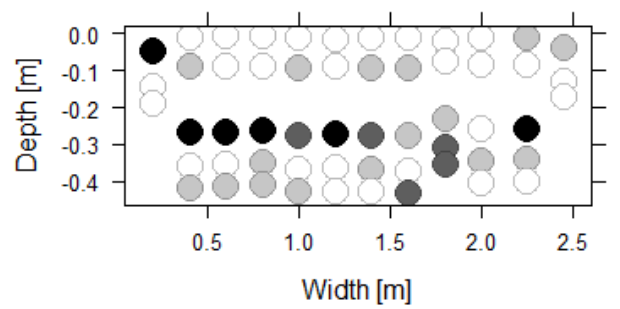

Inverse distance weighted

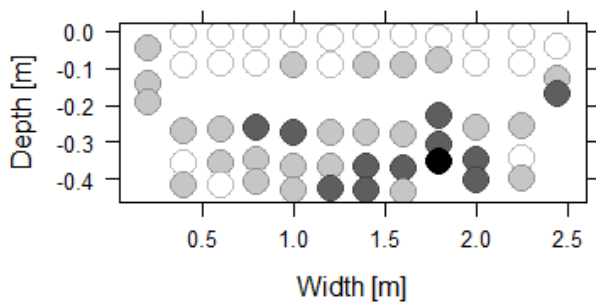

Ordinary kriging

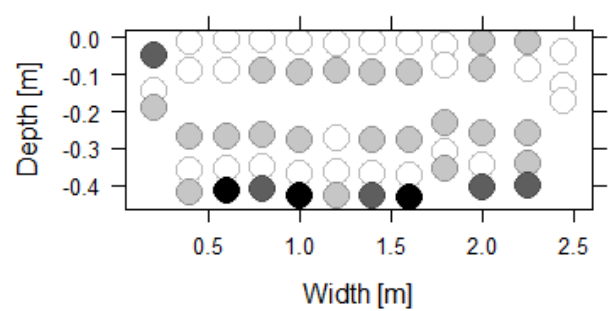

Fig. 4. Visualisation of the absolute error of velocities $\left[\mathrm{m} \cdot \mathrm{s}^{-1}\right]$ in the cross-validation in all estimated points in the validation data set 1.

\section{Arithmetical mean}

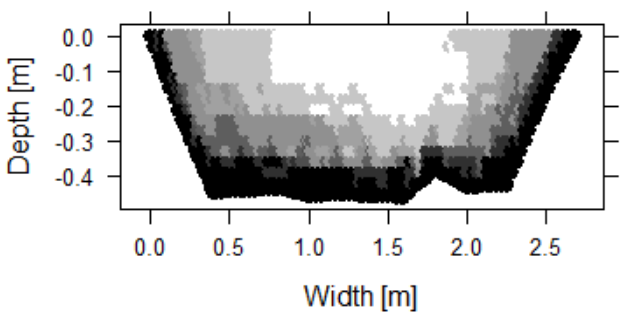

Thin-plate spline

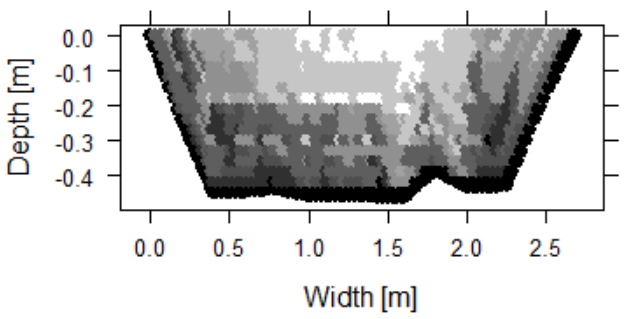

Inverse distance weighted

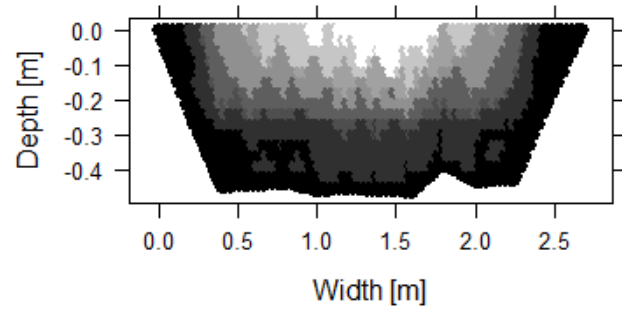

Ordinary kriging

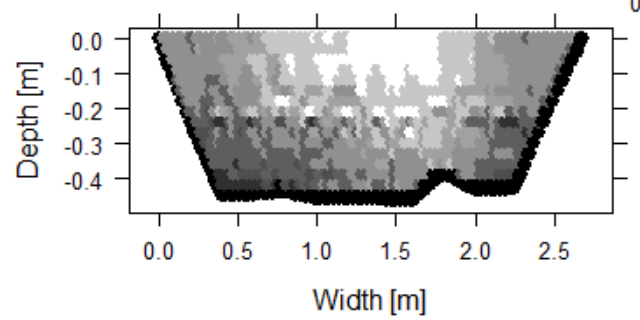

Fig. 5. Visualisation of interpolated velocities $\left[\mathrm{m} \cdot \mathrm{s}^{-1}\right]$ in a mesh of $3 \mathrm{~cm}$ in the validation data set 1.

Table 1. Mean values of the error criteria in absolute and relative form $\left[\mathrm{m} \cdot \mathrm{s}^{-1}\right]$ from the cross-validation of the validation data series for each method of interpolation computing.

\begin{tabular}{|c|c|c|c|c|c|c|c|c|}
\hline \multirow{2}{*}{$\begin{array}{l}\text { Computing } \\
\text { method }\end{array}$} & \multicolumn{8}{|c|}{ Error criterion $\left[\mathrm{m} \cdot \mathrm{s}^{-1}\right]$} \\
\hline & $\mathrm{ME}$ & $\mathrm{ME}_{\mathrm{R}}$ & MAE & $\mathrm{MAE}_{\mathrm{R}}$ & RMSE & $\mathrm{RMSE}_{\mathrm{R}}$ & STD & $\mathrm{STD}_{\mathrm{R}}$ \\
\hline $\begin{array}{l}\text { Arithmetic } \\
\text { mean }\end{array}$ & 0.039 & 0.118 & 0.058 & 0.18 & 0.081 & 0.315 & 0.071 & 0.293 \\
\hline $\begin{array}{l}\text { Inverse } \\
\text { distance } \\
\text { weighted }\end{array}$ & 0.055 & 0.177 & 0.066 & 0.21 & 0.088 & 0.361 & 0.066 & 0.313 \\
\hline $\begin{array}{l}\text { Thin-plate } \\
\text { spline }\end{array}$ & 0.036 & 0.13 & 0.081 & 0.266 & 0.118 & 0.505 & 0.111 & 0.488 \\
\hline $\begin{array}{l}\text { Ordinary } \\
\text { kriging }\end{array}$ & -0.003 & -0.066 & 0.076 & 0.26 & 0.102 & 0.452 & 0.101 & 0.45 \\
\hline
\end{tabular}

Note: ME - mean error, MAE - mean absolute error, RMSE - root mean squared error, STD - standard deviation of residuals. The criteria with the index $\mathrm{R}$ are in a relative form, the criteria without index $\mathrm{R}$ are in an absolute form. 
Table 2. Mean velocity $\left[\mathrm{m} \cdot \mathrm{s}^{-1}\right]$ of the validation data series and mean of the estimated values for each method of interpolation computing with percentage of the measured data in brackets.

\begin{tabular}{lccc}
\hline \multirow{2}{*}{ Computing method } & \multicolumn{2}{c}{ Mean velocity $\left[\mathrm{m} \cdot \mathrm{s}^{-1}\right]$} & and (percentage of mean of measured data) \\
\cline { 2 - 4 } & Valid. data set 1 & Valid. data set 2 & Valid. data set 3 \\
\hline Measured data set & 0.495 & 0.522 & 0.245 \\
\hline Arithmetic mean & $0.446(90)$ & $0.475(91)$ & $0.224(91)$ \\
\hline Inverse distance weighted & $0.412(83)$ & $0.471(90)$ & $0.214(87)$ \\
\hline Thin-plate spline & $0.447(90)$ & $0.498(95)$ & $0.209(85)$ \\
\hline Ordinary kriging & $0.505(102)$ & $0.521(100)$ & $0.245(100)$ \\
\hline
\end{tabular}

\section{DISCUSSION}

Interpolation in orthogonal mesh by IDW produced „bull's eyes", which does not agree with the general idea of a parabolic velocity distribution. The same phenomenon was for instance in Sanabria et al. (2013), Yao et al. (2013), Ünal and Özcakal (2011). The underestimation of velocities by IDW was another disadvantage in this case.

Higher values of the criteria computed in the validation process than the ones from the calibration process was caused by not completely covering variety in the data of the validation process by the calibration process. This situation is a common phenomenon if different data sets for calibration and validation are used. Neglectable growth of the error criterion between the calibration and the validation by the ordinary kriging was probably caused by fitting the semivariogram model on all available data sets. Optically more flat velocity profile made by the ordinary kriging corresponds with the result of Ünal and Özcakal (2011), who used Surfer software for the interpolation. In their paper, errors of IDW were higher than those of ordinary kriging, which is, except for ME, the opposite of the results presented here. Resulting RMSE in IDW is lower than the one in kriging in Yao et al. (2013), who used Geostatistical Analyst of ArcGIS software. Velocities estimated by the ordinary kriging were similar to measured ones in the presented paper, evaluated by the statistical pair test, and they had the same mean as the mean of measured velocities. This good result could be surprising, because using the ordinary kriging in the presence of apparent trend is arguable. But Journel and Rossi (1988) showed that estimations made by the ordinary kriging are unaffected by the trend specification. So they recommend simpler ordinary kriging for calculations also in case of clear possibility to split equation into a trend and use of more difficult kriging types with trend.

As Fig. 4 showed, more errors in the estimation by all methods occurred in the bottom and side parts of the crosssections. Positive correlation between MAE and location of estimated values reflects the right assumption to pay attention to a boundary condition. However, it does not seem to be enough. Adding more accurate adjustment with hydraulic boundary conditions and a calibration on more data sets could help.

The mean velocity persisting approximately equal calculated by all methods indicates that methods should be ready to use in 1-dimensional computing of discharge. With a well calibrated model, just a few measuring points from the field are needed and the same result is achieved as after a time-consuming measurement to obtain a number of measured points. But having a well calibrated model means having a simple tool for indication of places threatened with an erosion and a sedimentation, which is a multi-dimensional computing task. An enrockment on the correct part of a cross-section is economical and efficient.

\section{CONCLUSIONS}

Cross-validation of four interpolation models on several data sets from four cross-sections of three streams was made, including several calibration parameters. The thin-plate spline method reached the worst value of MAE and RMSE among used methods. The evaluation of velocity distribution in the orthogonal mesh was done, IDW produced unrealistic velocity profile. The shape of the velocity profiles interpolated using the model of arithmetic mean, thin-plate spline and ordinary kriging agreed with the general idea of a parabolic velocity distribution. Moderate positive correlation between depth and MAE was found for the arithmetic mean and IDW, more attention paid to the boundary layer could help improve the performance of the models. The best result in keeping the mean velocity was obtained using ordinary kriging.

Routine processing of the data measured with a propellertype current meter by standardized methods does not offer a direct assimilation of a spatial relation between the points measured in different verticals, which a spatial interpolation does. The estimated detail velocity distribution can be provided by the spatial interpolations.

Acknowledgement. The authors are grateful to the Grant Agenture of Faculty of Environmental Sciences of the Czech University of Life Sciences Prague (project No. 201342460) for the financial support and to Mr. Vondrů for the technical assistance in the field measurements.

\section{REFERENCES}

Baudin, M., Consortium Scilab - DIGITEO, 2011. Introduction to Scilab. Consortium Scilab - DIGITEO, URL http://forge.scilab.org/index.php/p/docintrotoscilab/downloa $\mathrm{ds} / 311 /$.

Bookstein, F., 1989. Principal warps: Thin-plate splines and the decomposition of deformations. IEEE Transaction on Pattern Analysis and Machine Intelligence, 11, 6, 567-585.

Clark, I., 2001. Practical Geostatistics. Geostokos limited, Alloa.

ČSN EN ISO 748, 2008. Hydrometrie - Měření průtoku kapalin $\mathrm{v}$ otevřených korytech použitím vodoměrných vrtulí a plováků. [Hydrometry - Measurement of liquid flow in open channels using current - meters or floats]. Český normalizační institut, Praha. (In Czech.)

Dyn, N., Levin, D., Rippa, S., 1986. Numerical procedures for surface fitting of scattered data by radial functions. Society for Industrial and Applied Mathematics, 7, 2, 639-659.

Fedorov, V., 1987. Kriging and other estimators of spatial field characteristics (with special reference to environmental studies): working paper. IIASA, Laxenburg. 
Journel, A.G., Rossi, M.E., 1988. When do we need a trend model? Study on statistics and environmental factors in health (SIMS). Stanford University, Stanford (California).

Madsen, H., van Griensven, A., Hojbjerk, A.L., 2010. Model calibration and validation in model-based water management. In: Vanrolleghem, P.A. (Ed.): Modelling Aspects of Water Framework Directive Implementation. IWA Publishing, London.

Raid, I., Kusnezowa, T., Seewig, J., 2013. Application of ordinary kriging for interpolation of micro-structured technical surfaces. Measurement Science \& Technology, 24, 9, 095201.

Sanabria, L.A., Qin, X., Li, J., Cechet, R.P., Lucas, C., 2013. Spatial interpolation of McArthur's Forest Fire Danger Index across Australia: Observational study. Environmental Modelling \& Software, 50, 37-50.

Starý, M., 2012. Personal communication by email on February 16.

Stošić, B.D., Silva, J.R.S., Filho, M.C., Cantalice, J.R.B., 2012. Optimizing river discharge measurements using Monte Carlo
Markov Chain. Journal of Hydrology, 450-451, 199-205.

Ünal, H.B., Özcakal, E., 2011. Determination of velocity dispersion profiles in open canals using different interpolation methods. Scientific Research and Essays, 6, 15, 3272-3280.

Venables, W.N., Smith, D.M., The R Core Team, 2014. An introduction to $\mathrm{R}$. The $\mathrm{R}$ Core Team. URL http://cran.rproject.org/doc/manuals/R-intro.pdf.

Wackernagel, H., 2003. Multivariate Geostatistics: an Introduction with Applications. Springer, Berlin.

Webster, R., Oliver, M.A., 2007. Geostatistics for Environmental Scientists. Second edition. John Wiley \& Sons Ltd, Chichester.

Yao, X., Fu, B., Lü, Y., Sun, F., Wang, S., Liu, M., 2013. Comparison of four spatial interpolation methods for estimating soil moisture in a complex terrain catchment. PLoS ONE, 8, 1, e54660.

Received 31 March 2014 Accepted 6 October 2014 